\title{
Path-independent J-integral for cracks in decagonal quasicrystals
}

\author{
Jan Sladek ${ }^{1, *}$, Vladimir Sladek ${ }^{1}$, and Miroslav Repka ${ }^{1}$ \\ ${ }^{1}$ Institute of Construction and Architecture, Slovak Academy of Sciences, 84503 Bratislava, Slovakia
}

\begin{abstract}
The path-independent $J$-integral is derived for fracture mechanics analysis of decagonal quasicrystals (QCs). The gradient theory of quasicrystals is developed here to consider large strain gradients at the crack tip vicinity. The constitutive equations contain phonon and phason stresses, and the higher-order stress tensor. The higher-order elastic material parameters are proportional to the internal length material parameter and the conventional elastic coefficients. The FEM equations are derived to solve general boundary value problems for the strain gradient theory of the QCs.
\end{abstract}

\section{Introduction}

The quasicrystals (QCs) have a structure of atoms as something between crystals and amourphous materials. It is observed a long-range quasiperiodic translational and orientational orders. They have special properties with effective engineering application. They were discovered in 1984 by Shechtman et al. [1]. The decagonal quasicrystals have ten-fold rotational symmetries and they belong to the class of two-dimensional (2-d) quasicrystals, with a quasiperiodic atomic arrangement in a plane, and a periodic one in the third direction.

In literature there are utilized three various models for a reliable description of elastodynamics of quasicrystals. Bak`s model [2] considers phasons for a particular structure disorders in quasicrystals. Then, phonons and phasons play similar roles in the dynamics and they are described by the balance of momentum. Lubensky et al. [3] consider that the phason field is described by a diffusion equation with a very large diffusion time. It follows from properties that phasons are insensitive to spatial translations and oppositely, phason modes represent the relative motion of the constituent density waves. Recently, Agiasofitou et al. [4] have utilized the wave - telegraph type equations for the elastodynamic model. However, a unique opinion on governing equations for phason fields is still missing. A comprehensive state of the art of investigations on the mechanical analyses of QCs can be found in monographs [5-7]. One can find there that QCs are generally considered as brittle materials. Therefore, crack analyses are very important to understand the effect of cracks on the mechanical behavior of a quasicrystal material. Up to now cracks are analyzed by classical elasticity theory [8-10].

\footnotetext{
*Corresponding author: jan.sladek@savba.sk
} 
It is well known that large strain gradients are occurred in the crack-tip vicinity. Therefore, the gradient elasticity theory appears most suited for studying the strain and stress fields near the crack-tip for crystalline materials [11-15]. In this study, the strain gradient theory is extended to quasicrystal materials. Constitutive equations in gradient theory of quasicrystals for phonon and phason stresses, and the higher-order stress tensor are given by the phonon and phason strains and the gradient of phonon strains in this study. The phason displacements represent already the atomic rearrangement and they are significantly smaller than phonon ones [16]. The gradients of phason strains are negligible in comparison to gradients of phonon strains, since the phason fields are smooth.

In this study, the higher-order $J$-integral and the energy release rate are derived for cracks in quasicrystals described by the strain-gradient elasticity. It is shown the path independence of this integral.

\section{Gradient theory of quasicrystals}

The elasticity theory of quasicrystals can be based on the phonon and phason displacements [5]. The phonon displacement $u_{i}(\mathbf{x})$ represents the phenomenological field corresponding to translational motion of atoms in crystals. Additional degrees of freedom corresponding to atomic rearrangements are introduced here. These are represented by the phason displacements $w_{i}(\mathbf{x})$. The phonon strains $\varepsilon_{i j}(\mathbf{x})$ of crystals are described by the gradients of phonon displacements

$$
\varepsilon_{i j}(\mathbf{x})=\frac{1}{2}\left(u_{i, j}+u_{j, i}\right),
$$

while the phason strains $w_{i j}(\mathbf{x})$ are defined as

$$
w_{i j}(\mathbf{x})=w_{i, j}(\mathbf{x}) \text {. }
$$

The constitutive equations for phonon and phason stresses in classical elasticity theory of quasicrystals have the following form $[5,16]$

$$
\begin{aligned}
& \sigma_{i j}=c_{i j k l} \varepsilon_{k l}+A_{i j k l} w_{k l} \\
& H_{i j}=K_{i j k l} w_{k l}+A_{k l i j} \varepsilon_{k l},
\end{aligned}
$$

where $c_{i j k l}, K_{i j k l}$ and $A_{k l i j}$ denote phonon elastic tensor, phason elastic tensor and phononphason coupling elastic constant tensor, respectively.

Since the phason strains are significantly smaller than phonon strains, the gradients of phason strains can be neglected in comparison with gradients of phonon strains in the gradient theory of quasicrystals. Then, the constitutive equations are written as

$$
\begin{gathered}
\sigma_{i j}=c_{i j k l} \varepsilon_{k l}+A_{i j k l} w_{k l}, \\
\tau_{j k l}=g_{j k l m n i} \eta_{m n i}, \\
H_{i j}=A_{k l i j} \varepsilon_{k l}+K_{i j k l} w_{k l},
\end{gathered}
$$

where $\mathbf{g}$ represents the higher-order elastic coefficients. The symbols $\tau_{i j k}$ are used to denote the higher-order stress tensor components.

The phonon strain-gradient tensor $\boldsymbol{\eta}$ is defined as

$$
\eta_{i j k}=\varepsilon_{i j, k}=\frac{1}{2}\left(u_{i, j k}+u_{j, i k}\right) .
$$

The constitutive equations for plane elasticity of decagonal QC are given by [5]

$$
\sigma_{11}=c_{11} \varepsilon_{11}+c_{12} \varepsilon_{22}+A\left(w_{11}+w_{22}\right),
$$




$$
\begin{gathered}
\sigma_{22}=c_{12} \varepsilon_{11}+c_{22} \varepsilon_{22}-A\left(w_{11}+w_{22}\right), \\
\sigma_{12}=\sigma_{21}=2 c_{66} \varepsilon_{12}+A\left(w_{21}-w_{12}\right), \\
H_{11}=K_{1} w_{11}+K_{2} w_{22}+A\left(\varepsilon_{11}-\varepsilon_{22}\right), \\
H_{22}=K_{1} w_{22}+K_{2} w_{11}+A\left(\varepsilon_{11}-\varepsilon_{22}\right), \\
H_{12}=K_{1} w_{12}-K_{2} w_{21}-2 A \varepsilon_{12}, \\
H_{21}=K_{1} w_{21}-K_{2} w_{12}+2 A \varepsilon_{12},
\end{gathered}
$$

where material coefficients $c_{i j}, A$ and $K_{i}$ denote the classical phonon elastic coefficients, the phonon-phason coupling parameter and the phason elastic coefficients, respectively.

In gradient theory, these constitutive equations can be extended according to (4) and rewritten as

$$
\begin{aligned}
& {\left[\begin{array}{l}
\sigma_{11} \\
\sigma_{22} \\
\sigma_{12}
\end{array}\right]=\left[\begin{array}{ccc}
c_{11} & c_{12} & 0 \\
c_{12} & c_{22} & 0 \\
0 & 0 & c_{66}
\end{array}\right]\left[\begin{array}{c}
\varepsilon_{11} \\
\varepsilon_{22} \\
2 \varepsilon_{12}
\end{array}\right]+\left[\begin{array}{cccc}
A & A & 0 & 0 \\
-A & -A & 0 & 0 \\
0 & 0 & -A & A
\end{array}\right]\left[\begin{array}{l}
w_{11} \\
w_{22} \\
w_{12} \\
w_{21}
\end{array}\right]=} \\
& =\mathbf{C}\left[\begin{array}{c}
\varepsilon_{11} \\
\varepsilon_{22} \\
2 \varepsilon_{12}
\end{array}\right]+\mathbf{A}\left[\begin{array}{c}
w_{11} \\
w_{22} \\
w_{12} \\
w_{21}
\end{array}\right] \\
& {\left[\begin{array}{l}
H_{11} \\
H_{22} \\
H_{12} \\
H_{21}
\end{array}\right]=\left[\begin{array}{cccc}
K_{1} & K_{2} & 0 & 0 \\
K_{2} & K_{1} & 0 & 0 \\
0 & 0 & K_{1} & -K_{2} \\
0 & 0 & -K_{2} & K_{1}
\end{array}\right]\left[\begin{array}{l}
w_{11} \\
w_{22} \\
w_{12} \\
w_{21}
\end{array}\right]+\left[\begin{array}{ccc}
A & -A & 0 \\
A & -A & 0 \\
0 & 0 & -A \\
0 & 0 & A
\end{array}\right]\left[\begin{array}{c}
\varepsilon_{11} \\
\varepsilon_{22} \\
2 \varepsilon_{12}
\end{array}\right]=} \\
& =\mathbf{A}^{\mathbf{T}}\left[\begin{array}{c}
\varepsilon_{11} \\
\varepsilon_{22} \\
2 \varepsilon_{12}
\end{array}\right]+\mathbf{K}\left[\begin{array}{c}
w_{11} \\
w_{22} \\
w_{12} \\
w_{21}
\end{array}\right], \\
& {\left[\begin{array}{c}
\tau_{111} \\
\tau_{221} \\
\tau_{121} \\
\tau_{112} \\
\tau_{222} \\
\tau_{122}
\end{array}\right]=l^{2}\left[\begin{array}{cccccc}
c_{11} & c_{13} & 0 & 0 & 0 & 0 \\
c_{13} & c_{33} & 0 & 0 & 0 & 0 \\
0 & 0 & c_{66} & 0 & 0 & 0 \\
0 & 0 & 0 & c_{11} & c_{13} & 0 \\
0 & 0 & 0 & c_{13} & c_{33} & 0 \\
0 & 0 & 0 & 0 & 0 & c_{66}
\end{array}\right]\left[\begin{array}{c}
\eta_{111} \\
\eta_{221} \\
2 \eta_{121} \\
\eta_{112} \\
\eta_{222} \\
2 \eta_{122}
\end{array}\right]=l^{2} \mathbf{G}\left[\begin{array}{c}
\eta_{111} \\
\eta_{221} \\
2 \eta_{121} \\
\eta_{112} \\
\eta_{222} \\
2 \eta_{122}
\end{array}\right], \quad \mathbf{G}=\left[\begin{array}{cc}
\mathbf{C} & \mathbf{0} \\
\mathbf{0} & \mathbf{C}
\end{array}\right] .}
\end{aligned}
$$

In equation (9) it is assumed that higher-order elastic parameters $g_{j k l m n i}$ are proportional to the conventional elastic stiffness coefficients $c_{k l m n}$ as $g_{j k l m n i}=l^{2} \delta_{l i} c_{j k m n}$ and $l$ is the internal length material parameter [17]. 
The density of the deformation energy of quasicrystal solids in gradient elasticity [15, 16] becomes

$$
\mathrm{U}(\varepsilon . ., w . ., \eta \ldots)=\frac{1}{2} c_{i j k l} \varepsilon_{k l} \varepsilon_{i j}+\frac{1}{2} K_{i j k l} w_{k l} w_{i j}+A_{i j k l} w_{k l} \varepsilon_{i j}+\frac{1}{2} l^{2} c_{i j m n} \eta_{m n k} \eta_{i j k} .
$$

Then, the variation of deformation energy in a solid with volume $V$ is given by

$$
\begin{aligned}
\delta U & =-\int_{V}\left[\left(\sigma_{i j, j}-\tau_{i j k, j k}\right) \delta u_{i}+H_{i j, j} \delta w_{i}\right] d V+\int_{\Gamma}\left[n_{j}\left(\sigma_{i j}-\tau_{i j k, k}\right) \delta u_{i}+n_{k} \tau_{i j k} \delta u_{i, j}+n_{j} H_{i j} \delta w_{i}\right] d \Gamma= \\
= & -\int_{V}\left[\left(\sigma_{i j, j}-\tau_{i j k, j k}\right) \delta u_{i}+H_{i j, j} \delta w_{i}\right] d V+\int_{\Gamma}\left\{t_{i} \delta u_{i}+R_{i} \delta s_{i}+Q_{i} \delta w_{i}\right\} d \Gamma
\end{aligned}
$$

where $s_{i}:=\frac{\partial u_{i}}{\partial \mathbf{n}}, R_{i}:=n_{k} n_{j} \tau_{i j k}, Q_{i}:=n_{j} H_{i j}$ and the traction vector is defined as

$t_{i}=n_{j}\left(\sigma_{i j}-\tau_{i j k, k}\right)-\frac{\partial \rho_{i}}{\partial \boldsymbol{\pi}}+\sum_{c}\left\|\rho_{i}\left(\mathbf{x}^{c}\right)\right\| \delta\left(\mathbf{x}-\mathbf{x}^{c}\right)$

$\rho_{i}:=n_{k} \pi_{j} \tau_{i j k}$

with $\pi_{i}$ being the Cartesian components of the unit tangent vector on $\Gamma$.

The external work of the applied external "forces" $\left(\bar{t}_{i}, \bar{R}_{i}, \bar{Q}_{i}\right)$ is given by

$$
\delta W=\int_{\Gamma_{t}} \bar{t}_{i} \delta u_{i} d \Gamma+\int_{\Gamma_{R}} \bar{R}_{i} \delta s_{i} d \Gamma+\int_{\Gamma_{Q}} \bar{Q}_{i} \delta w_{i} d \Gamma .
$$

From the principle of virtual work, viz., $\delta U-\delta W=0$, the following governing equations is obtained:

$\sigma_{i j, j}(\mathbf{x})-\tau_{i j k, j k}(\mathbf{x})=0$,

$H_{i j, j}(\mathbf{x})=0$.

\section{$3 \mathrm{~J}$-integral for quasicrystals}

At a virtual extension of a crack along its plane by $d a$, the change of potential energy $\Pi$ in cracked quasicrystal body $\Omega$ is given by

$$
\begin{aligned}
\frac{\mathrm{d} \Pi}{\mathrm{d} a} & =\int_{\Omega} \frac{\mathrm{dU}}{\mathrm{d} a} d \Omega-\int_{\partial \Omega_{t}} \bar{t}_{i} \frac{\mathrm{d} u_{i}}{\mathrm{~d} a} d \Gamma-\int_{\partial \Omega_{R}} \bar{R}_{i} \frac{\mathrm{d} s_{i}}{\mathrm{~d} a} d \Gamma-\int_{\partial \Omega_{Q}} \bar{Q}_{i} \frac{\mathrm{d} w_{i}}{\mathrm{~d} a} d \Gamma= \\
& =\int_{\Omega} \frac{\mathrm{dU}}{\mathrm{d} a} d \Omega-\int_{\partial \Omega}\left(t_{i} \frac{\mathrm{d} u_{i}}{\mathrm{~d} a}+R_{i} \frac{\mathrm{d} s_{i}}{\mathrm{~d} a}+Q_{i} \frac{\mathrm{d} w_{i}}{\mathrm{~d} a}\right) d \Gamma .
\end{aligned}
$$

Futhermore it is assumed that crack grows along $x_{1}$ axis of the coordinate system and its origin moves together with the crack-tip. The following identity is valid

$$
\frac{\mathrm{d}}{\mathrm{d} a}=\frac{\partial}{\partial a}+\frac{\partial x_{1}}{\partial a} \frac{\partial}{\partial x_{1}}=\frac{\partial}{\partial a}-\frac{\partial}{\partial x_{1}} .
$$


That identity is utilized in eq. (16)

$$
\frac{\mathrm{d} \Pi}{\mathrm{d} a}=\int_{\Omega}\left(\frac{\partial \mathrm{U}}{\partial a}-\frac{\partial \mathrm{U}}{\partial x_{1}}\right) d \Omega-\int_{\partial \Omega}\left[t_{i}\left(\frac{\partial u_{i}}{\partial a}-\frac{\partial u_{i}}{\partial x_{1}}\right)+R_{i}\left(\frac{\partial s_{i}}{\partial a}-\frac{\partial s_{i}}{\partial x_{1}}\right)+Q_{i}\left(\frac{\partial w_{i}}{\partial a}-\frac{\partial w_{i}}{\partial x_{1}}\right)\right] d \Gamma .
$$

Since $\delta \mathrm{U}=\frac{\partial \mathrm{U}}{\partial a}, \delta W=\frac{\partial W}{\partial a}$, and utilizing the principle of virtual work, equation (18) is reduced to

$$
\frac{\mathrm{d} \Pi}{\mathrm{d} a}=-\int_{\Omega} \frac{\partial \mathrm{U}}{\partial x_{1}} d \Omega+\int_{\partial \Omega}\left(t_{i} \frac{\partial u_{i}}{\partial x_{1}}+R_{i} \frac{\partial s_{i}}{\partial x_{1}}+Q_{i} \frac{\partial w_{i}}{\partial x_{1}}\right) d \Gamma .
$$

Applying the Gauss divergence theorem to domain integral in eq. (19), one can write

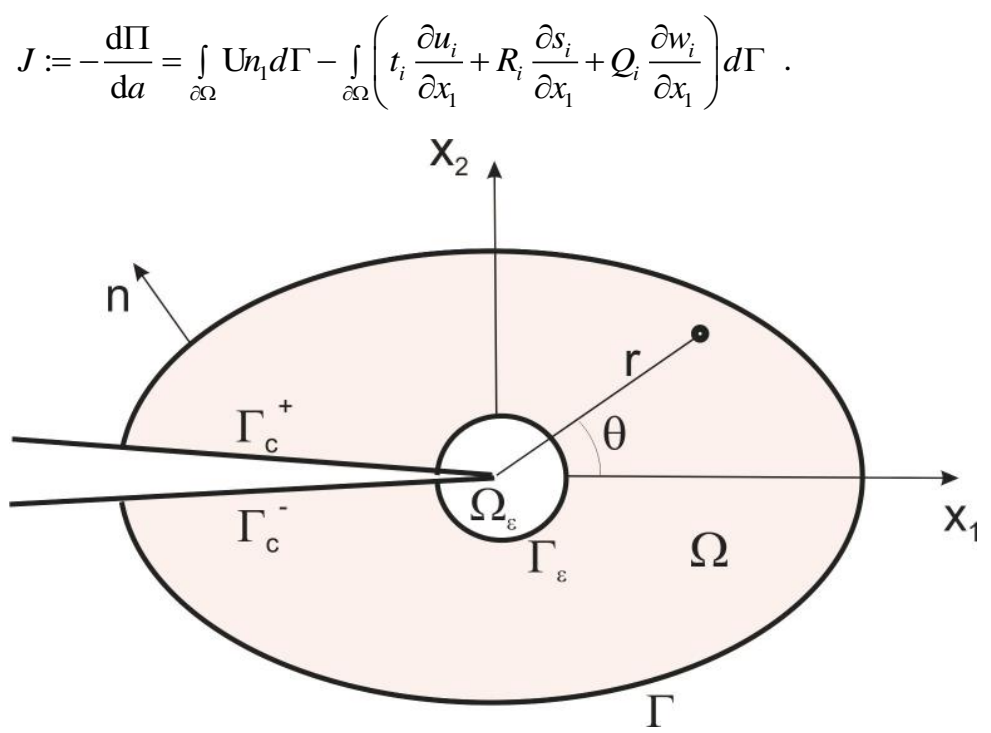

Fig. 1. Integration paths and coordinate definitions.

Next, it is shown that $J=0$, if the domain $\Omega$ does not contain any irregular point. The irregular point at the crack tip is omitted by the domain $\Omega_{\varepsilon}$ in Fig. 1 . Then, the shaded zone is regular domain with boundary $\partial \Omega=\Gamma+\Gamma_{c}^{+}+\Gamma_{c}^{-}-\Gamma_{\varepsilon}$. Since $\mathrm{U}=\mathrm{U}\left(\varepsilon_{i j}, \eta_{i j k}, w_{i j}\right)$,

$$
\begin{aligned}
& \frac{\partial \mathbf{U}\left(\varepsilon_{i j}, \eta_{i j k}, w_{i j}\right)}{\partial x_{1}}=\frac{\partial \mathrm{U}}{\partial \varepsilon_{i j}} \frac{\partial \varepsilon_{i j}}{\partial x_{1}}+\frac{\partial \mathrm{U}}{\partial \eta_{i j k}} \frac{\partial \eta_{i j k}}{\partial x_{1}}+\frac{\partial \mathrm{U}}{\partial w_{i j}} \frac{\partial w_{i j}}{\partial x_{1}}=\sigma_{i j} \frac{\partial u_{i, j}}{\partial x_{1}}+\tau_{i j k} \frac{\partial u_{i, j k}}{\partial x_{1}}+H_{i j} \frac{\partial w_{i, j}}{\partial x_{1}}= \\
& =\left(\sigma_{i j}-\tau_{i j k, k}\right) \frac{\partial u_{i, j}}{\partial x_{1}}+\left(\tau_{i j k} \frac{\partial u_{i, j}}{\partial x_{1}}\right)_{, k}+H_{i j} \frac{\partial w_{i, j}}{\partial x_{1}}= \\
& =\left[\left(\sigma_{i j}-\tau_{i j, k}\right) \frac{\partial u_{i}}{\partial x_{1}}\right]_{, j}+\left(\tau_{i j k} \frac{\partial u_{i, j}}{\partial x_{1}}\right)_{, k}+\left(H_{i j} \frac{\partial w_{i}}{\partial x_{1}}\right)_{, j},
\end{aligned}
$$

in which the governing equations (15) are utilized. 
If eq. (21) is utilized in eq. (20) we get that J-integral is vanishing in a regular domain

$$
\begin{aligned}
& J=\int_{\Omega}\left\{\left[\left(\sigma_{i j}-\tau_{i j k, k}\right) \frac{\partial u_{i}}{\partial x_{1}}\right]_{, j}+\left(\tau_{i j k} \frac{\partial u_{i, j}}{\partial x_{1}}\right)_{, k}+\left(H_{i j} \frac{\partial w_{i}}{\partial x_{1}}\right)_{, j}\right\} d \Omega-\int_{\partial \Omega}\left(t_{i} \frac{\partial u_{i}}{\partial x_{1}}+R_{i} \frac{\partial s_{i}}{\partial x_{1}}+Q_{i} \frac{\partial w_{i}}{\partial x_{1}}\right) d \Gamma= \\
& =\int_{\partial \Omega}\left\{n_{j}\left(\sigma_{i j}-\tau_{i j k, k}\right) \frac{\partial u_{i}}{\partial x_{1}}+n_{k} \tau_{i j k} \frac{\partial u_{i, j}}{\partial x_{1}}+n_{j} H_{i j} \frac{\partial w_{i}}{\partial x_{1}}\right\} d \Omega-\int_{\partial \Omega}\left(t_{i} \frac{\partial u_{i}}{\partial x_{1}}+R_{i} \frac{\partial s_{i}}{\partial x_{1}}+Q_{i} \frac{\partial w_{i}}{\partial x_{1}}\right) d \Gamma= \\
& =\int_{\partial \Omega}\left\{\left(\frac{\partial \rho_{i}}{\partial \boldsymbol{\pi}}-\sum_{c}\left\|\rho_{i}\right\| \delta\left(\mathbf{x}-\mathbf{x}^{c}\right)\right) \frac{\partial u_{i}}{\partial x_{1}}+n_{k} \tau_{i j k} \frac{\partial u_{i, j}}{\partial x_{1}}-n_{k} n_{j} \tau_{i j k} \frac{\partial}{\partial \mathbf{n}} \frac{\partial u_{i}}{\partial x_{1}}\right\} d \Gamma= \\
& =\int_{\partial \Omega}\left\{-n_{k} \pi_{j} \tau_{i j k} \frac{\partial}{\partial \boldsymbol{\pi}} \frac{\partial u_{i}}{\partial x_{1}}-n_{k} n_{j} \tau_{i j k} \frac{\partial}{\partial \mathbf{n}} \frac{\partial u_{i}}{\partial x_{1}}+n_{k} \tau_{i j k} \frac{\partial u_{i, j}}{\partial x_{1}}\right\} d \Gamma= \\
& =\int_{\partial \Omega}\left\{-n_{k} \tau_{i j k} \frac{\partial u_{i, j}}{\partial x_{1}}+n_{k} \tau_{i j k} \frac{\partial u_{i, j}}{\partial x_{1}}\right\} d \Gamma \equiv 0 .
\end{aligned}
$$

Since $\partial \Omega=\Gamma+\Gamma_{c}^{+}+\Gamma_{c}^{-}-\Gamma_{\varepsilon}$, Eq. (20) can be written as

$J=J_{0}+J_{c}^{+}+J_{c}^{-}-J_{\varepsilon}=0$,

where

$$
\begin{aligned}
& J_{0}=\int_{\Gamma}\left(U n_{1}-t_{i} \frac{\partial u_{i}}{\partial x_{1}}-R_{i} \frac{\partial s_{i}}{\partial x_{1}}-Q_{i} \frac{\partial w_{i}}{\partial x_{1}}\right) d \Gamma, \\
& J_{\varepsilon}=\int_{\Gamma_{\varepsilon}}\left(U n_{1}-t_{i} \frac{\partial u_{i}}{\partial x_{1}}-R_{i} \frac{\partial s_{i}}{\partial x_{1}}-Q_{i} \frac{\partial w_{i}}{\partial x_{1}}\right) d \Gamma, \\
& J_{c}^{ \pm}=\int_{\Gamma_{c}^{ \pm}}\left(U n_{1}-t_{i} \frac{\partial u_{i}}{\partial x_{1}}-R_{i} \frac{\partial s_{i}}{\partial x_{1}}-Q_{i} \frac{\partial w_{i}}{\partial x_{1}}\right) d \Gamma .
\end{aligned}
$$

Since the crack is along $x_{1}$, i.e. $\left.n_{1}\right|_{\Gamma_{c}^{ \pm}}=0$, and assuming the natural boundary conditions on $\Gamma_{c}^{ \pm}$on both crack faces, we can see that $J_{c}^{ \pm}=0$. Then, according to (23), the $\mathrm{J}$-integral on an arbitrary integration path $J_{0}$ is equal to $J_{\varepsilon}$. It means that J-integral is path independent.

\section{Numerical example}

In this section, an edge crack in a finite, square body under uniform phonon tension load on top of the strip, $\bar{t}_{2}=\sigma_{0}=1 \mathrm{~Pa}$ is analyzed. Due to the symmetry with respect to $x_{1}-$ axis, only a half of the strip is modeled. This is shown in Fig. 2 with the appropriate boundary conditions. The following geometric parameters are considered: $w=5.0 \times 10^{-7} \mathrm{~m} a=w / 8$. 


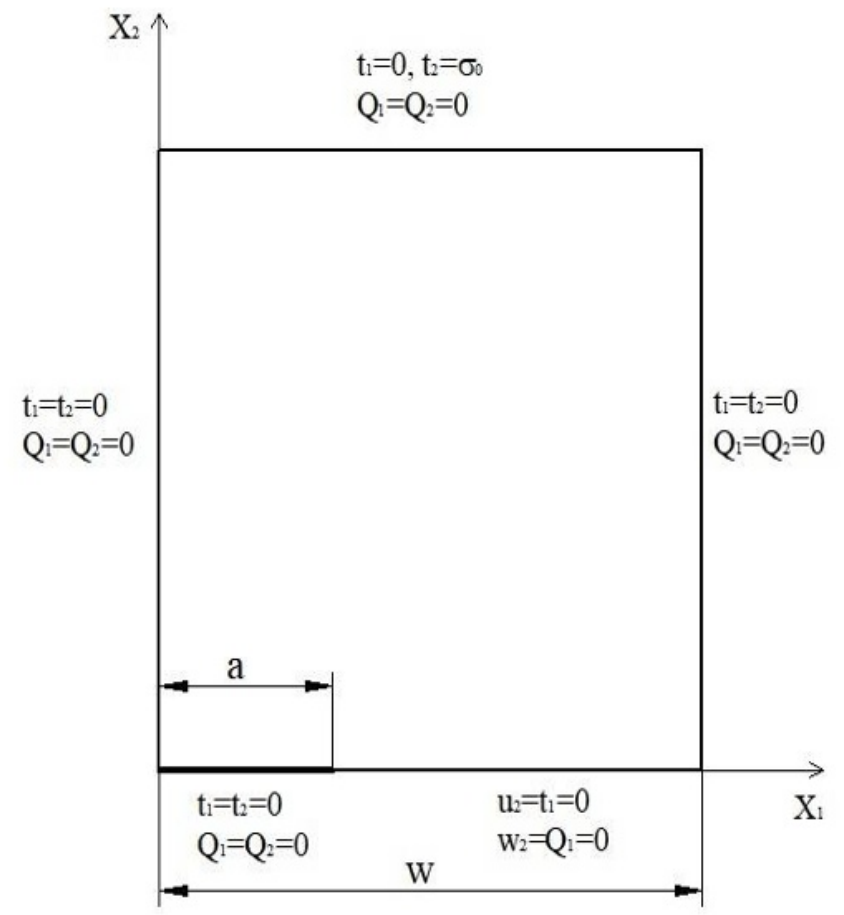

Fig. 2. Boundary conditions for the edge crack in a finite square plate.

The following material parameters corresponding to decagonal quasicrystal are considered $[5]$

$$
\begin{aligned}
& c_{11}=L+2 M=23.43 \cdot 10^{10} \mathrm{Nm}^{-2}, \quad c_{12}=L=5.74 \cdot 10^{10} \mathrm{Nm}^{-2}, \quad K_{1}=12.2 \cdot 10^{10} \mathrm{Nm}^{-2}, \\
& K_{2}=2.4 \cdot 10^{10} \mathrm{Nm}^{-2}, \quad M=\left(c_{11}-c_{12}\right) / 2, \quad A=1.1 \cdot 10^{9} \mathrm{Nm}^{-2} \\
& \rho=4180 \mathrm{~kg} / \mathrm{m}^{3}, \quad \Gamma_{w}=4.8 \cdot 10^{-19} \mathrm{~m}^{3} \mathrm{~s} / \mathrm{kg} .
\end{aligned}
$$

The analyzed domain is discretized by 7227 elements. If the internal length material parameter $l$, is vanishing the theory is reduced to standard classical QC results. To show the influence of this material parameter on $J_{0}$ integral, there are considered various values of $l$ in numerical analyses and they are represented by the strain-gradient size-factor $q$, as

$l^{2}=q \cdot l_{0}^{2}$,

where $l_{0}=5 \times 10^{-9} \mathrm{~m}$ is chosen [15].

If the ratio $l /(a / w)$ is decreasing both phonon and phason crack displacements are reduced with respect to ones obtained by classical theory. A similar phenomenon is observed for $J_{0}$ integral. A reduction of the $J_{0}$ integral value with growing the size effect parameter is observed in Fig. 3. Similar behaviour has been observed also for crystal material [15]. 


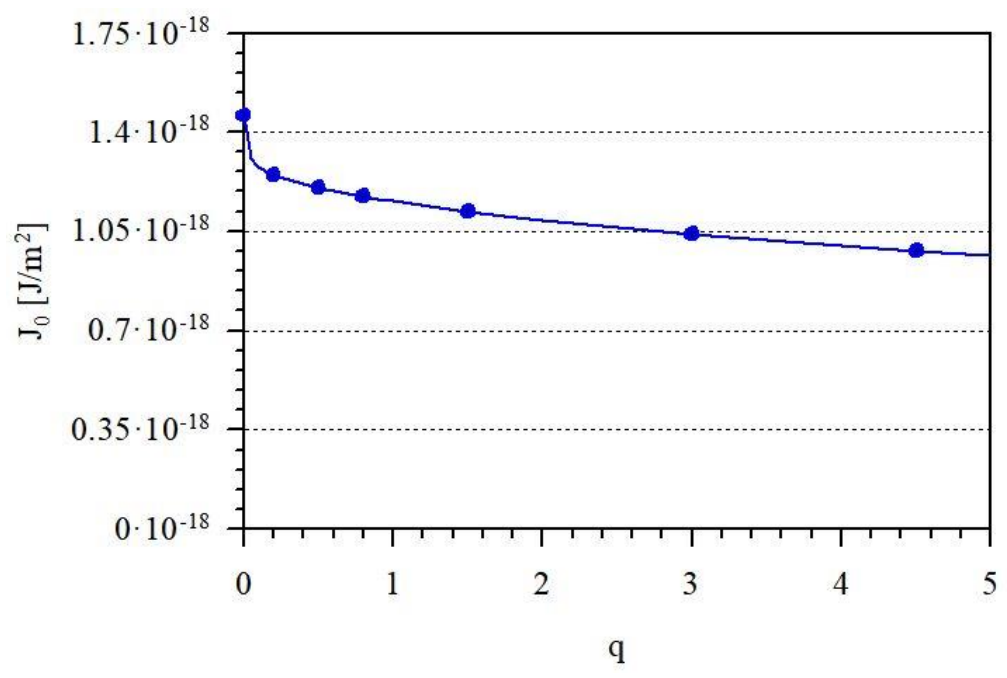

Fig. 3. Variation of the $J_{0}$ integral with the length-scale parameter q.

\section{Conclusion}

The $J$-integral defined in a global coordinate system vanishes when the integration contour chosen to calculate the $J$-integral encloses all irregularities in considered domain. The $J_{o}$ integral obtained in this paper will provide a useful way to study fracture problems in quasicrystal materials.

The authors acknowledge the support of the Slovak Science and Technology Assistance Agency registered under number APVV-18-0004, and VEGA-2/0046/16.

\section{References}

1. D. Shechtman, I. Blech, D. Gratias, J.W. Cahn, Phys. Rev. Lett. 53, 1951-1953 (1984)

2. P. Bak, Phys. Rev. Lett. 54, 1517-1519 (1985)

3. T.C. Lubensky, S. Ramaswamy, J. Joner, Phys. Rev. B 32, 7444-7452 (1985)

4. E. Agiasofitou, M. Lazar, Int. J. Solids Struct. 51, 923-929 (2014)

5. T.Y. Fan, Mathematical theory of elasticity of quasicrystals and its applications (Springer, Berlin, 2011)

6. T.Y. Fan, Mathematical theory of elasticity of quasicrystals and its applications (second edition, Springer, Berlin, 2016)

7. T.Y. Fan, Generalized dynamics of soft-matter quasicrystals-Mathematical mpodels and solutions (Springer, Berlin, 2017)

8. X.F. Li, T.Y. Fan, Y.F. Sun, Phil. Magazine A 79, 1943-1952 (1999)

9. X.F. Li, T.Y. Fan, Appl. Math. Comput. 196, 1-5 (2008)

10. Y. Li, M.H. Zhao, Q.H. Qin, C.Y. Fan, Appl. Math. Modelling 69, 648-664 (2019)

11. I. Vardoulakis, G. Exadaktylos, E. Aifantis, Int. J. Solids Struct. 33, 4531-4559 (1996) 
12. G. Exadaktylos, I. Vardoulakis, E. Aifantis, Int. J. Fracture 79, 107-119 (1996)

13. N. Aravas, A.F. Giannakopoulos, Int. J. Solids Struct. 46, 4478-4503 (2009)

14. Y. Wei, Eur. J. Mechanics A/Solids 25, 897-913 (2006)

15. J. Sladek, V. Sladek, P. Stanak, Ch. Zhang, C.L. Tan, Int. J. Solids Struct. 113, 1-9 (2017)

16. J. Sladek, V. Sladek, S. Krahulec, Ch. Zhang, M. Wunsche, Int. J. Fracture 181, 115$126(2013)$

17. I. Gitman, H. Askes, E. Kuhl, E. Aifantis, Int. J. Solids Struct. 47, 1099-1107 (2010) 